\title{
MAPPING THE PROGRESS IN AGILE PRODUCT DEVELOPMENT: A MULTI-CASE STUDY
}

\author{
Schmidt, Tobias Sebastian (1); Behrenbeck, Jan (2); Burger, Kevin (2); Hostettler, Rafael (2); \\ Paetzold, Kristin (1); Zimmermann, Markus (2) \\ 1: University of the German Federal Armed Forces Munich; 2: Technical University of Munich
}

\begin{abstract}
The more dynamic and unpredictable the development constraints, the more agile the development project should be to cope with and utilize inherent change. Especially in such contexts, aligning with the project's mission and vision, committing to next steps, and documenting the development path is challenging. To support the decision making process of self-organized agile development teams with an overview, a recent research paper proposes the Progress Map. The investigation at hand applies the Progress Map in semi-industrial development projects to empirically validate its applicability and performance in the form of a multi-case study. The results indicate that, given future refinements, this instrument can be valuable to plan, document, and communicate the progress of an agile development project.
\end{abstract}

Keywords: Design management, Design practice, Case study, Agile Development in Mechatronics, Knowledge management

\section{Contact:}

Schmidt, Tobias Sebastian

University of the German Federal Armed Forces Munich

Institute of Technical Product Development

Germany

tobias.schmidt@unibw.de 


\section{INTRODUCTION}

Trends such as globalization, digitization and technological progress lead to more complex, dynamic and unpredictable conditions for mechatronic product development. These changes require an increased reactivity and adaptability of engineers and development teams (Baltes et al., 2017). In software development these conditions are tackled using agile development approaches. According to Böhmer et al. (2015) "agility is the capability to react, and adopt to expected and unexpected changes within a dynamic environment constantly and quickly; and to use those changes [...] as an advantage." This ability is crucial to survive under ever-changing conditions (Bennet et al., 2014).

According to the Agile Manifesto documentation has a minor priority in agile development (Beck et al., 2001). Therefore, agile methods come with their own form of documentation, or are agnostic to the specific implementation (e.g. user stories can be collected on post-its just as well as in an spread sheet or requirements engineering tool). Still, experience shows there is an apparent lack in documentation especially of the meta process. A quick oversight how the project has been progressing over time, which hypotheses have been abandoned and why and how decisions have been taken is usually not available. Similarly to that retrospective view, upcoming activities are often determined spontaneously so that agile development is considered chaotic in planning. Especially in an agile setting, where decisions are dense and changes in direction frequent, having such an overview readily available is valuable not only for research and analysis purposes, but also to on-board new team members, communicate the process to stakeholders or structure project planning. For that, Schmidt et al. (2018a) propose the Progress Map that bases on Concept/Knowledge-Theory (CK-Theory), set-based design, and the repository tree from software development. Although its strengths have been described in theory, the Progress Map lacks a comprehensive validation in practice. Thus, the study answers the following research question:

\section{Is the Progress Map empirically valid? What are strengths and weaknesses of the Progress Map when applied in agile development projects?}

In order to analyze the applicability and performance of the Progress Map in semi-industrial agile development projects, the initial concept was integrated into The Agile Framework (TAF), an agile method for mechatronic product development, and applied at Think.Make.Start (TMS), a two-weeks lab course on product design and innovation at the Technical University Munich (Böhmer et al., 2016; Hostettler and Böhmer, 2017). A multi-case study supported by qualitative interviews was carried out.

\section{STATE OF THE ART}

\subsection{Agile development at a glance}

The field of agile development consists of the methodological philosophy as well as guiding methods and practices. In the so called Manifesto of Agile Software Development, 4 values and 12 principles form the philosophy, i.e., the overarching paradigm of what is important and how to deal with certain situations in agile development (Beck et al., 2001). It frames the way of thinking to increase the corporate competitiveness under ever-changing development constraints (Schmidt et al., 2018b). Correspondingly, perceiving change as chance to learn and to utilize it for one's own benefit instead of hedging against the negative consequences (e.g., risk management) is fundamental to agile development (Böhmer et al., 2015). To do so, an agile development project is incremental and iterative in nature (Oestereich and Weiss, 2007). In each iteration, the project team realizes a new artifact that is used for learning and unveiling the unforeseen (Oestereich and Weiss, 2007). In this way, the project team gradually learns how to perform better and aligns its future project activities accordingly.

To make the philosophy of agile development easily applicable in daily business, Scrum, e.g., provides a process and role model that structures the project organization (Schwaber and Sutherland, 2017). However, depending on the project context, it is recommended to choose an appropriate agile method such as eXtreme Programming, Kanban or Design Thinking. To best fit the context of TMS being the case study context of subsequent investigation, Hostettler and Böhmer (2017) derived TAF which combines best practices from various agile methods under a single process model that is specifically adapted to the conditions of TMS.

TAF relies on the aspects of successful innovations according to Design Thinking being feasibility (does the concept technically work?), desirability (does the concept attract some customer segments?) and viability (is the concept economically reasonable in the long-term?). TAF suggests to iterate in 
these domains to test hypotheses by various means. E.g., the project team can conduct interviews with potential users to test desirability, build paper prototypes to check the look and feel, and construct functional prototypes to prove feasibility. TAF does not hinge on a specific task management framework and works with Kanban or Scrum boards alike, in the TMS context mostly Kanban is used. TAF's central aim is to reduce risks with as little resources as possible and therefore seeks quick prototypes, which can be rough, but usually imply important insights for the next iteration. Therefore, in contrast to Scrum where a prototype is due every 1 to 4 weeks, TAF cycles are not fixed in length and are usually very short (hours) in the beginning, growing in length as complexity increases. Furthermore, TAF does not contain a role model that specifies sets of tasks and responsibilities team members should fulfill. Especially, there is no product owner to allow user iteration for every team member (Hostettler and Böhmer, 2017).

\subsection{CK-Theory and set-based design}

The CK-Theory explains the process of design by means of two expandable spaces. On the one hand, the knowledge space summarizes all propositions that are known for sure. It represents the current knowledge base of the team. On the other hand, the concept space consists of design concept propositions that are at least partially uncertain, i.e., the proposition has no logical status in the knowledge space. In both spaces, propositions build up and depend on each other so that the spaces expand during development as new design concepts evolve in the concept space (new design ideas) and insights are gained in the knowledge space (validated learning) (Hatchuel and Weil, 2003; Agogué and Kazakçi, 2014).

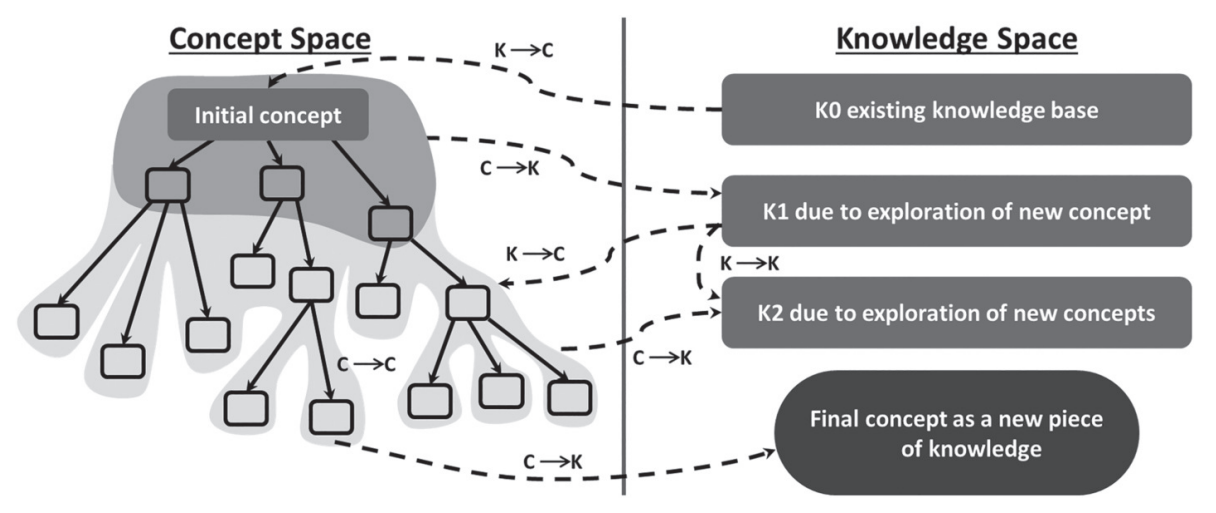

Figure 1. CK-Theory explained (Agogué and Kazakçi, 2014)

As visualized in Figure 1, the CK-Theory allows design explorations to different directions $(C \rightarrow C)$ and an advancement by logical reasoning within the knowledge space $(K \rightarrow K)$, but fosters reflection to the knowledge base $(C \rightarrow K)$ which again stimulates new design concept $(K \rightarrow C)$ propositions (Agogué and Kazakçi, 2014). By exploring new grounds, the CK-Theory supports the logic of set-based design in which a set of design concepts is developed further in parallel (Sobek et al., 1999). As soon as new knowledge is available to the team, decisions whether to cut off less potent concepts can be made while remaining as flexible as possible to cope with future change (Reinertsen, 2009). In contrast to pointbased design, which allows only a single valid design concept at a time, set-based design has substantial value for agile development (Stelzmann, 2011).

\subsection{Progress measurement in agile development}

To measure the progress of an agile development team especially in mechatronics, Schmidt et al. (2018a) suggest the Progress Map which bases primarily on CK-Theory and set-based design. As illustrated in Figure 2, the Progress Map contains promoters that symbolize fields of high potential in regard to the project vision (what to deliver?) and mission (why to deliver?). Promoters can be, e.g., user stories that state the customer value and, thus, deliberately leave the design of the feature open. Starting at the bottom, the map builds up in an iterative manner while the team keeps on exploring and exploiting the promoters. As each iteration results in new insights, the team gets closer to the vision successively.

Future iterations are subject to planning and past iterations show the documented development path. That is because in each iteration the team reflects on potential future development directions based on the current knowledge base and commits itself on a few directions to be focused in the upcoming 
iteration. Key insights gained during the iteration are finally attached to the map and linked to both previous knowledge propositions and the prototype that led to the new insight. In that way, the map builds up towards the top, i.e., the project vision and mission (Schmidt et al., 2018a).

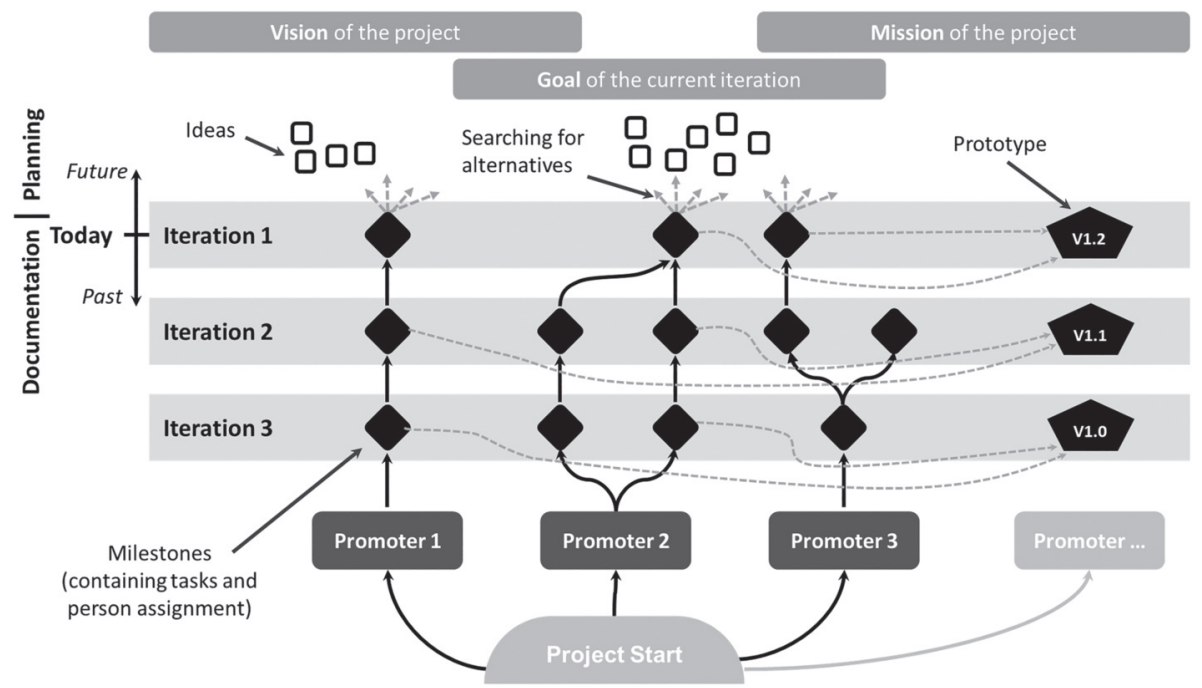

Figure 2. Basic idea of the progress map (Schmidt et al., 2018a)

To complete the tool landscape of TAF in terms of both documentation and planning activities, a variant of the Progress Map was a prudent approach to provide a better overview of (past and future) project activities. This Progress Map variant is called Tobi.Board and is specifically adapted to TAF. The promoters were fixed to the three dimensions (desirability, feasibility, and viability) of TAF. An iteration consisted of one plan-do-check-act (PDCA) cycle, where several plan-do-check (PDC) sub-cycles could be run in parallel and in a joint "act" phase a decision was taken how to progress further. Each PDC subcycle would be represented by a card, containing a plan, do, and check section, as well as an indicator as to which method would be applied throughout this cycle. The "plan" section consisted of the hypothesis or open question to be answered, the "do" section contained all the documentation accrued during the evaluation and the "check" section an aggregated answer to the hypothesis. Additionally a pivot-marker could be set to indicate failed hypotheses. Since this was the central tool to see the progress of the team, three boxes that describe the overall vision, mission, and goal were added as visualized in Figure 3.
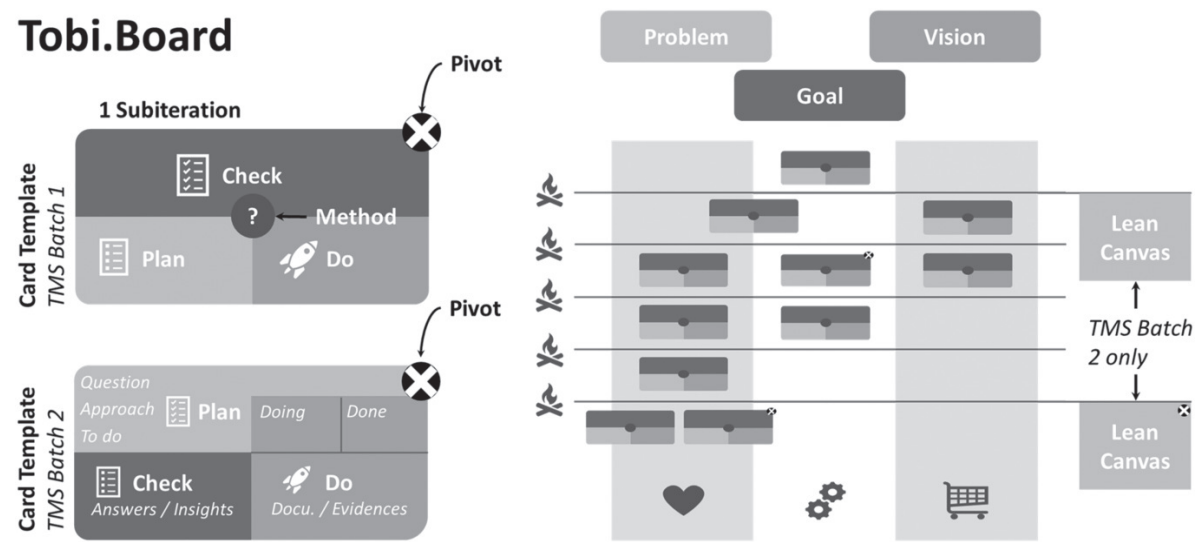

Figure 3. Concept of Tobi.Board

\section{RESEARCH APPROACH}

\subsection{Expected value proposition of the Tobi.Board}

The presented concept of the board is theoretically expected to support agile project teams in three aspects of both project planning and documentation: Firstly, the board might support the teams in planning the content of tasks by prioritizing open questions, hypotheses, and features in order to focus 
on the project's most critical aspects (desirability, feasibility, and viability). By focusing the available resources on the most relevant questions, the time and financial investment risk is minimized.

Secondly, the Tobi.Board is supposed to support the organizational planning of the project by linking responsibilities and liabilities (such as deadlines or resources) to individual task packages in iteration cycles. In particular, the integration of Kanban post-its into cards is meant to allow an overview of the current work status at any time. This might not only promote communication and coordination between team members but also facilitate project and resource management. The integration of organizational details into cards during the planning phase is expected to help identifying knowledge carriers in the event of later queries, thus, also to make tacit knowledge usable. This information on knowledge distribution could then be used for efficient personnel allocation for future work packages.

In addition to the planning benefits, advantages in project documentation and knowledge management are expected. The cards might enable team members to systematically record their content results, working documents and explicit knowledge in a clear and structured way, yet with sufficient context-specific freedom while spending minimum time as the cards have already been prepared during the planning phase. Since new tasks build on completed and documented previous tasks, the development path and design rationales should be traceable for everyone at any time. This is supposed to help avoiding conflicts, misunderstandings and dead ends or - in case of problems - to find the source, identify false friends knowledge and incorrect assumptions. The possibility to fall back on past solution stages leads to an increased degree of planning reliability and ensures requirement/assumption traceability. Planning and documentation might thereby benefit from one another.

For clarification it needs to be mentioned at this point, that the board has no intention of measuring the progress or interpreting results in the context of the overall systems design. Its only purpose is to map the progress to support planning and documentation. It is the project team's responsibility to frequently reflect on the context in the "act" phases to ensure that the project objectives (vision, mission) are met.

\subsection{Application setting}

The Tobi.Board was applied by students in a semi-industrial lab course called TMS at the Technical University Munich. TMS is a two-weeks cross-faculty block course, in which Master's students are challenged to identify real problems in their environment, form interdisciplinary teams, ideate on possible solutions, evaluate their ideas, and test their hypotheses with prototypes and by talking to potential customers (Böhmer et al., 2016). Every team independently works on their own project.

Although it is an educational setting, students need to apply for TMS, that takes place in a business incubator, to select participants with an entrepreneurial mindset (acceptance rate of about 20\%). The predominating purpose of the course is to apply the broad engineering and management knowledge they gained in their studies to real market problems and to preferably start-up a new business on the developed product as problem solution. For that, TAF gives methodical guidance but grants extensive freedom in selecting situation-adequate approaches. Being close to graduation, receiving credits and grades for systematically approaching and using appropriate tools is perceived less attractive by the bulk of the students than having the opportunity to establish a start-up in a systematic and guided manner. Technological and methodical coaching by experienced mentors as well as a comprehensive and modern infrastructure (3D printers, laser cutters, etc.) play a crucial role. Thus, TMS can be considered as a very specific, but a semi-industrial product development context.

The Tobi.Board was tested by $\mathbf{2 4}$ teams in two successive batches of the course in mid 2018. The student feedback from batch 1 was evaluated and incorporated into a slightly modified board design which was then used in batch 2. The first version consisted of an adaptation of the Progress Map only. The second version integrated Kanban as a more detailed task management system and more space for documentation in the "do" section as visualized in Figure 3. Additionally the boxes 'Problem', 'Goal', and 'Vision' were substituted by a Lean Canvas, that was filled and edited while moving along with iterations and insights.

The first course had 43 participants from 9 departments forming 10 groups of 3 to 5 students each. The second course had 70 participants from 7 departments forming 14 teams of 5 students each. The majority of students was in their Master's and had no to few experience with TAF. The student teams were asked to use the board for task planning and for documentation of their findings. In daily team progress presentations students used the board in the plenum to present and discuss their insights. 


\subsection{Explanatory research design}

The investigation was based on a multi-case study of 24 TMS projects from 2 batches supported by explorative interviews. 3 of the authors were involved as TMS coach so that the study follows a participatory action research approach. Thus, the authors did not observe the teams only, but were involved in terms of teaching and advising. One author was a TMS participant, whose involvement was limited on a single case only, to include the user point of view to the case analyses.

The cloud-based software application Real Time Board (now called Miro: www.realtimeboard.com), that allows for collaborative editing of an object-based digital whiteboard, was used as a tool to create and visualize the team boards. All Tobi.Boards of each batch were accumulated in one large document. Every morning, a back-up copy of the document was generated to analyze the daily changes. However, during batch 2 the board was deleted twice during the course due to technical problems which made students create analogous substitutes manually.

After both TMS batches, 25 TMS students (15 from batch 1, 10 from batch 2) and 3 coaches were interviewed in order to collect qualitative feedback about the use and application of the Tobi.Board. The interviews were conducted personally either face-to-face or via Skype to thoroughly understand the reasoning. The questionnaire based on open-ended questions and fostered narrative conversations. The interviews were conducted after grading in order to prevent student bias towards what they might think the interviewer wants to hear. To explore the reasoning from different perspectives, the questionnaire was adapted in an iterative manner. In total, three interview iterations were made, each lasting a week. While the first iteration included no prepared questionnaire, the second iteration questionnaire build upon the insights gained in the first iteration. Similarly, the questionnaire was advanced for the third iteration. While the first iteration was deliberately open, the last iteration focused on confirming the trends identified before (iteration 1: 9x batch 1 students; iteration 2: $4 \mathrm{x}$ batch 1 and $6 \mathrm{x}$ batch 2 students; iteration 3: $2 \mathrm{x}$ batch $1,4 \mathrm{x}$ batch 2 students and $2 \mathrm{x}$ coaches).

\section{FINDINGS}

\subsection{Example case}

As introducing all 24 cases would be too much, a representative case was selected. The team LOCI developed a smart shopping cart for grocery stores to (a) increase consumer satisfaction by reduced search time via intelligent indoor routing and (b) to generate comprehensive consumer data for advanced heat map analysis which can be used for product placement optimization and personalized advertisement. However, they initially intended to improve navigation of passengers on large airports and train stations because they have experienced the problem themselves. Within 15 (PDCA) iterations and 37 (PDC) subiterations, LOCI learned, e.g., that there exist solutions on the market already, that consumers at grocery stores desire a way to reduce search time, but grocery stores nor consumers want to pay for existing solutions being very complex, and that data about the consumer is very valuable for grocery stores. Each of the 37 subiterations represent a card on the Tobi.Board that was filled with the open question to be answered (e.g., "Do consumers prefer a flashing shopping cart or augment reality glasses for navigation?"), the method how to answer the question (e.g., interviews with paper prototypes), the documented answers (e.g., link to Google Drive with interview recordings and pictures) and the conclusion on the question (e.g., "most consumers prefer a cart indicating the way"). 10 of these cards and their underlying assumption were pivoted leading to important insights and dead ends on the Tobi.Board.

The entire team met 2 to 3 times a day to review what are new insights, new challenges and new opportunities (act phase), and to discuss what is still uncertain or unknown and what to do next (plan phase). Having formulated new open questions, which the team cannot answer yet, but are important to proceed towards the increasingly sharpening mission and vision, the team divided in subteams which each committed on answering a single card by the end of the iteration.

Having visualized their development path on the Tobi.Board made it obvious for LOCI that they are strong in desirability, but lack feasibility and viability because less activities were present in these promoters until the 8th iteration. They started to consider more thoroughly the remaining promoters and finally proved by several prototypes that their product idea is technically feasible, desired by a specific market segment to a large extent and potentially viable so that it would make sense to continue the project in the form of a start-up. 


\subsection{Qualitative evaluation}

The narrative interviews revealed that, in general, the basic tenor is positive among all interviewees and most students state that they would use the concept again even outside of TMS. However, some problems were reported and are primarily focused in the following.

Most teams understood and used the Tobi.Board primarily as a tool for documentation. In a two weeks course, however, many results or decisions were still remembered by the team members and there was only a minor need for documentation. Many mentioned though, that in longer lasting projects this would definitely be more relevant. Additionally, it took some time for the teams to learn which information to put where (confusing according to some students). In both batches, the card template structure (plan, do, check) was not intuitive as found by some teams. One of the coaches interpreted this difficulty as a consequence of generally not being used to documentation rather than an actual issue with the board.

Nevertheless, the teams perceived the approach of asking clear questions or stating hypotheses and having to answer them before moving to the next iteration very valuable for ideation. Especially in the very early stages of the development when the idea for the project was not settled yet, the need for asking specific questions resulted in focus and clarity. Participants reported that without this guidance they would have been very uncertain about where to start. A great advantage of the concept.

Most teams iterated once per day and other teams managed to iterate 2-3 times per day which also includes 2-3 (rough) prototypes per day. Interestingly, the fast-iterating teams were better organized and less stressed in achieving their goals than others according to the coaches. Most of them assigned a "Kanban master" or a Scrum master, who took care of task management. Iterations were handled in two different ways. Either the teams worked in a time-boxed manner (fixed duration, variable output) or in goal-driven manner (variable duration, fixed output). However, none of both options could be identified as better or worse than the other.

The crash of the Real Time Board in batch 2 led to teams switching to "offline mode". A physical board on the wall, mostly Kanban-centered, was the reaction of many. One team decided to take photos of all boards at the end of each day in order to have a simple documentation of their project path. This revealed to be less effort but fulfilled the same need for progress documentation.

The separation of the Tobi.Board into desirability, feasibility, and viability was commonly perceived as very helpful. Although some students reported that they would have liked to categorize their work themselves, others commented that it helped them to approach the problem holistically and, therefore, made sure no aspect was overseen. Most teams naturally separated into engineers working on feasibility, industrial designers on desirability and business students on viability.

The board layout induced to start at the bottom and move upwards which provided difficulties for the teams. "Moving left-to-right and up-to-down is the natural way we do things" as one participant said. Moreover, the necessity of zooming in to fill the cards and zooming out to get the overview was a shortcoming to the often-praised benefit of providing great transparency. In contrast, there was not enough space in one box of the cards (in the "do" field) for many participants in order to document everything. However, some admitted that the card template forced the team to document only key insights.

As initially stated, one PDCA cycle can include multiple PDC subiterations, i.e., parallel iterations of sub-teams. This tends to overload the board and it becomes unclear which insight led to which hypothesis. For that reason, some teams introduced arrows to show causal connection of cards over the course of multiple iterations. Working on different cards in parallel made the teams more efficient and more solidly grounded in terms of the overall validity of their product concept.

The teams reported that the guided PDCA-cycle brought a lot of structure and focus to development. Summing up and reflecting on the insights of each PDCA-cycle in a few words in the "check" section of the Tobi.Board cards results in both clarity and transparency. But both batches had difficulties with the right sequence of P-D-C as it was not intuitive (especially in batch 1 the sequence was written counterclockwise on the card), a simple numbering (1. Plan, 2. Do, 3. Check) would have helped. Furthermore the "method" field was perceived redundant to "do". The "approach" field in batch 2 turned out to be an improvement here, as it leaves room for own words. In general, the card template structure of batch 2 was evaluated more intuitive, but the batch 1 card templates grants more freedom so that it seems that students favor the batch 1 card structure.

Many teams relied on Kanban as their main productivity technique. As batch 1 had difficulties with too many tools at multiple places (Kanban physically on the wall, lean canvas on another wall, Tobi.Board 
digitally) an adjusted layout of the Tobi.Board for batch 2 suggested Kanban as part of the boxes in the digital Tobi.Board. But only one team of batch 2 reported to actually having used it for a while. Everyone else switched to a big physical Kanban on the wall for the following reasons. Firstly, having it physical on post-its in clear sight on the wall feels more intuitive and present. Secondly, most students were confused by having the tasks attached to the hypothesis cards which, in turn, are scattered.

While batch 1 worked with an "offline" lean canvas, consisting of tape and post-its on the wall, for batch 2 an evolving lean canvas was introduced into the digital Tobi.Board. A new product or business idea required to update or refill the canvas while old versions were stored digitally attached to the corresponding iteration. There was no clear trend in terms of which approach was received better by the users. One of the coaches reported that, by having to create a completely new version of the canvas for each new business concept the users were "forced to ask all questions again" (coach A).

The greatest value of the Tobi.Board was seen in the aspect of documentation. At every stage it is possible to see what has been done, what is in progress and what can be the next steps. Therefore, it is also possible to jump back in time when "dead ends", i.e., development routes without a promising future, are met without having to fear that things are done twice. This also motivated almost all teams because progress was explicitly visible. Visual documentation is also very helpful when there is the necessity to regularly report the progress of the project. In the context of TMS this was done by daily progress presentations. Furthermore, having a "not-too-rigid and not-too-flexible guideline in the 'wild' early stage" (student D, batch 2) of an agile product development project was perceived as highly valuable. Having to ask specific questions and remain working on them until an answer was found, provides a robust guideline. But also the need to iterate quickly, i.e., following the PDCA-cycle was of great value. Because of these benefits, more than half of the interviewed persons stated that they would use the Tobi.Board again. However they suggest three changes. First, the software must be more reliable or the Tobi.Board should be completely offline. Secondly, they would only use it for longer projects with a higher need for documentation. Thirdly, they would use it especially for (partly) distributed teams where documentation online ensures that all team members are up-to-date.

\section{DISCUSSION}

\subsection{Concept refinement}

The original idea of the Progress Map has been adapted to the setting of TMS and applied in 24 teams distributed over two batches. Experiences gained from the first batch influenced the board and card template as well as recommended daily working routines for the second batch. Having analyzed the 24 cases it became obvious that the Progress Map concept includes some hidden logical "breaking lines" that separate three modules. Mostly, these modules are applicable independently and, hence, create possibilities to configure the Progress Map as required due to contextual constraints.

On the one hand, the planning module (module 1) contains the upper part of the board, i.e., the future perspective. It is about collecting ideas for promising future development paths continuously and reflecting on them in light of the problem to be solved, mission to be aligned at and goal to be achieved in the next iteration. However, module 1 can differentiate between organizational planning and product planning. While the latter deals with what and how to do, the former covers the who, when and by which means. Although organizational planning depends on product planning, product planning could be done without considering organizational planning.

On the other hand, module 2 is about documenting the development paths including key insights and reasons why certain directions were pursued or not. Card designs as shown in Figure 3 perform very well for documenting explicit knowledge. Tacit knowledge that is difficult or even impossible to articulate can be considered by adding the persons that were involved in the corresponding PDCA-cycle. In this way, project members cannot only reconstruct key insights gained and how they were derived, but also who might have further tacit knowledge and could support in case of future questions.

Furthermore, module 3 deals with accompanying documents. These can be of various kinds such as morphological box or lean canvas, and advance in line with the project progress. Their corresponding design or business concept could be pivoted, refined, updated or restructured. For the sake of documentation, it seems valuable to attach these document versions to the Progress Map to enrich the development path visualization and complement reasons why to choose certain directions. 


\subsection{Recommended use of the Tobi.Board}

Since Real Time Board crashed multiple times especially in the second batch and made it impossible for the teams to continue with the digital Tobi.Board template, most of them imitated the template with post-its, paper cards, whiteboards, and tape. Although unintended, the Real Time Board downtime provided the chance to investigate the differences in applying the Tobi.Board in an online versus offline mode.

Concerning the planning module, using post-its is very intuitive because it is tangible and present. Based on the experience from TMS, this makes it easier to teach the concept of Tobi.Board compared to an online implementation. However, if the team cannot work in the same room (e.g., because subteams conduct field tests or interviews elsewhere), a digital implementation is more appropriate because accessibility serves as a key parameter.

When it comes to documentation, a digital implementation is definitely adequate since it is easily expandable and allows a higher degree of freedom, e.g., to link other documents directly with the cards. Nevertheless, the combination of Tobi.Board and Real Time Board for the sake of documentation does not work well because Real Time Board is cumbersome and laborious in navigating and creating content in the given structure. Compulsorily, an analogous implementation of the documentation was tested with post-its, paper, and tape. But as expected, documenting on a wall comes with repositioning the items often, which is unproductive. Furthermore, an analogous board is extendable to a limited degree so that documentation after a certain number of iterations cannot be continued.

Similarly, implementing accompanying documents in the Tobi.Board analogously or digitally depends on the expected rate of change, need for mobile accessibility, and expandability. Especially when implemented digitally, the version history can be mapped more neatly.

As a consequence, Tobi.Board implementations depend on the project-specific circumstances and should be decided jointly within the team. Research can only give guidance by providing selection strategies to choose the most appropriate combination under given contextual constraints. Generally speaking, if the team wants to apply the planning module solely, it should be done analogously. On the contrary, if the team wants to apply the documentation module only, it should be done digitally. If the team wants to use both modules, implementing the planning module analogously and the documentation module digitally is not recommended because planning prepares documentation and can propel project productivity. In this case it seems more appropriate to favor a digital solution. Finally, module 3 correlates directly with the documentation module and should be implemented accordingly.

\subsection{Benefits for an agile project team}

Based on the findings the expected value of Tobi.Board can be evaluated. The first value which is support in planning the content of tasks was confirmed by a majority of students. The board helped them to focus the available resources on the most relevant questions and to frequently consider the larger context of their work. Many teams used lines and arrows to connect different tasks to improve traceability. This helped them to communicate and interpret their overall progress with respect to their overall project objectives. Many students stated that they want to use this aspect of the board in future projects.

The second expected benefit being organizational planning of the project could only be confirmed to some extent. In general, the tool supported teams in managing their resources and the integration of Kanban post-its into cards allowed an overview of the current work status. But it also increased complexity which, in some cases, led to confusions as the board was not updated regularly or consistently. The students reported the positive benefits of Kanban in project management and that a link between the cards and the Kanban post-its would be useful but that an integration into the digital tool reduces the usability of Kanban significantly. Most teams preferred using a physical Kanban board as an extension to the progress board. As the project only lasted two weeks and teams did not mix, the expected benefit of making tacit knowledge usable and personnel allocation easier could not be evaluated.

Project documentation and knowledge management was found to be very well supported by the Tobi.Board. They find the structure and the aspects to be filled out very helpful and highly relevant for agile product development because the development path becomes traceable. Especially in everchanging conditions, having a tool at hand to transparently support decision making was stated to have significant value for TMS and for self-organized development teams in general. 


\subsection{Limitation}

However, in the context of TMS, the relevance of result documentation for information sharing is diminished as (a) the projects last only 2 weeks, inducing a low need for comprehensive documentation, and (b) the participants are collocated to most of the time and involved in one project only, allowing for fast and direct communication and knowledge transfer. These aspects represent distinct differences to a real industrial project and, thus, limit the validity of the investigation. Product development in industry concerns also large and distributed project teams, a multitude of stakeholder interests as well as high process and product complexity. In this light, TMS is a simplified semi-industrial, but riskless context in which preliminary testing of new design science artifacts (such as the Progress Map) is insightful and valuable before applying it to more complex industrial settings. Hence, the study has a preliminary characteristic with a limited generalizability to industrial development projects, but pushes new methodical ideas into practice.

\section{SUMMARY AND CONCLUSION}

In order to validate the applicability and value of the Progress Map proposed by Schmidt et al. (2018a) the investigation adapted the concept to the setting of TMS and applied the so derived Tobi.Board to 24 project teams. It turned out that the Tobi.Board is most valuable especially when it comes to documenting the development paths. It provides a structured and intuitive way to keep an overview of all project activities in the past and future, but lacks acceptance for planning in many cases. Nevertheless, the validity of the Progress Map concept is empirically proven to a large extent as most expected benefits were observable indeed, although the study has a preliminary nature from an industry point of view. The implementation of the Progress Map strongly depends on the specific project context. Based on the multi-case study it seems wise to let the project team decide which modules of the Progress Map are appropriate as the team itself knows the contextual constraints best. Frequent team retrospectives can fine-tune the adaptation by critical reflections. The modular structure of the map as described allows a more flexible selection of what really matters and is of value for the specific project. More work needs to be done particularly in regards to industry implementation.

\section{REFERENCES}

Agogué, M. and Kazakçi, A. (2014), "10 Years of C-K Theory”, In: Chakrabarti, A. and Blessing, L.T.M. (Ed.), An Anthology of Theories and Models of Design, Springer, London, pp. 219-235. https://doi.org/10.1007/978-1-4471-6338-1

Baltes, G. and Selig, C. (2017). “Organisationale Veränderungsintelligenz”, In: Baltes, G. and Freyth, A. (Ed.), Veränderungsintelligenz, Springer Gabler, Wiesbaden, pp. 81-168. https://doi.org/10.1007/978-3-658-04889-1_2

Beck, K., Beedle, M., Bennekum, A. van, Cockburn, A., Cunningham, W., Fowler, M. et al. (2001), Manifesto for Agile Software Development. [online]. Available at: agilemanifesto.org (30 November 2018).

Bennett, N. and Lemoine, G.J. (2014). "What a difference a word makes: Understanding threats to performance in a VUCA world", Business Horizons, Vol. 57 No. 3, pp. 311-317. https://doi.org/10.1016/j.bushor.2014.01.001

Böhmer, A., Beckmann, A. and Lindemann, U. (2015), “Open Innovation Ecosystem - Makerspaces within an Agile Innovation Process", ISPIM Innovation Summit, Brisbane, Australia.

Böhmer, A.I., Richter, C., Hostettler, R., Schneider, P., Plum, I., Böhler, D. et al. (2016). “Think.Make.Start. - An Agile Framework", 14th International Conference on Engineering Design, pp. 917-926.

Hatchuel, A. and Weil, B. (2003), "A new approach of innovative design: An introduction to C-K theory", 14th International Conference on Engineering Design, pp. 109-125.

Hostettler, R. and Böhmer, A.I. (2017), "Reducing Uncertainty within Minimum Time and Resources", 23th International ICE Conference on Engineering, Technology and Innovation, Madeira, Portugal.

Oestereich, B. and Weiss, C. (2007), APM - Agiles Projektmanagement, dpunkt.verlag, Heidelberg.

Reinertsen, D. (2009), The Principles of Product Development Flow: Second Generation Lean Product Development, Celeritas Publishing, Redondo Beach.

Schmidt, T.S., Gerdzhikov, G. and Paetzold, K. (2018a), "Set-based Design in Agile Development: Reinterpreting the Repository Tree", International Conference on Engineering, Technology and Innovation, Stuttgart.

Schmidt, T.S., Weiss, S. and Paetzold, K. (2018b), "Expected vs. Real Effects of Agile Development of Physical Products Apportioning the Hype", International Design Conference, Dubrovnik, Croatia, pp. 2121-2132. https://doi.org/10.21278/idc.2018.0198

Schwaber, K. and Sutherland, J. (2017), The Scrum Guide, Scrum.Org and ScrumInc, p. 19.

Sobek Ii, D.K., Ward, A.C. and Liker, J.K. (1999), “Toyota's principles of set-based concurrent engineering”, MIT Sloan Management Review, Vol. 40 No. 2, pp. 67-83.

Stelzmann, E.S. (2011), Agile Systems Engineering: Eine Methodik Zum besseren Umgang mit Veränderungen bei der Entwicklung komplexer Systeme, Ph.D. Thesis, Technical University Graz, Austria. 